INH A L T

Zu diesem Brind

ABHANDLUNGEN, STUDIEN, MISZELLEN

Zu den Themenkrelsen des VI. Internatlonalen Kongresegs der WIrt8chaft shtstoriker 1974 in Kopenhagen

R. Borthold

M. Nussbaum

H. Mottek

W. Schmidt

D. Goetz

P. Khalatbart

J. Kahk/E. Tarvel

M. A. Barg

W. Strenz/H. Thümmle:

K. - R. Biermann
Der soz Lalökonomische DlfferenzlerungsprozeB der Bauernw trt Bchaft in der Provinz Brandenburg whenrend der industriellen Revolution (1816 bls 1878/82)

Unternehmenskonzentration und Investstrategle nach dem ersten Weltkrleg. Zur Eutwicklung des deutschen Groskapitals whinrend und nach der groBen Inflation unter besonderer Berlicksichtigung der Schwertndustrie

Wirtschaftsgeschichte und Umwelt

Zu den Bezlehungen zwlschen dem UrbanlslerungsprozeB und der Herausbildung des Inneren Marktes In Indien

Naturwissenschaftliche Aspelte der deutschen Aufklärung. Zur naturwissenschaftlichen Blldung an den Universituten

Zu den Bezlehungen zwischen Llandern mit unterschledlichem Entwicklungsstand aus historischer Sicht. Bezlehungen zwischen Entwicklungsländern und hocbentwickelten kapttalistl schen Landern

Zwel Versuche zur Anwendung mathematischer Methoden in der agrargeschlchtlichen For schung

Dle Arbeltsprodultivitut in der Landwirtschaft Im England des 11. bls 13. Jahrhunderts, un ter besonderer Bertickslchtlgung der methodischen Aspelte des Forschungegegenstandes

Zur Problemattk der Erarbeltung von Strelkkarten

Alexander von Humboldt als Mlinzreformer 
J. Kahk

P. Hess

G. Heltz

\section{BETRIE BSGESCHICHTE}

H. O. Gericke
Eine Zwlschenbilanz der Anwendung mathematischer Methoden in der sowjetischen Geschichts wissenschaft. Bemerkungen zum Forschungsstand im Zusammenhang mit Matematiceskie metody $\vee$ IstoriCeskich issledovanijach. Sbornik statej

Die historisch-theoret ische Methode in ihrer Anwendung auf die Entwicklungsländer. Zu einem schöpferischen Versuch. Parviz Khalatbari, Ökonomische Unterentwicklung

Agrargeschichtliche Probleme eines Histori schen Ortslexikons

Die Unterstiutzung der Betriebsgeschichts schreibung durch die Kommission zur Erfor schung der Geschichte der örtlichen Arbeiterbewegung bei der Bezirksleitung der SED Magdeburg

\section{TAGUNGEN UND KONFERENZEN}

Vierte Jahrestagung des interdlsz iplinären Arbeitskollektivs zur Erforschung der Geschichte der Produktivkräfte

K. Lärmer Tagungsbericht

W. Jonas

Thesen zum Wesen der Industriellen Revolution

K. Spading

17. Jahrestagung der Hansischen Arbeitsgemeinschaft der Historiker-Gesellschaft der DDR vom 10. bis 12 . Oktober 1972 in Frankfurt (Oder)

M. Straube

"Haupttendenzen der europäischen Stadtgeschichte". Arbeitstagung vom 23. bis 24. Oktober 1972 in Magdeburg

P. Wick

"Geschichte und Computer", eine internationale Konferenz vom 25. bis 29. Juni 1973 in Uppsala (Schweden)

J. Peters

Beratung des Jahrbuchs für Wirtschaftsgeschichte iber internationale Zusammenarbeit am 25 . und 26. September 1973 in Berlin 


\section{QUELLEN UND MATERIALIEN}

Bestandsinformation für den Wirtschaft shistoriker aus dem Zentralen Staatsarchiv Potsdam

K. Metschies

Der Bestand Deutsche Revisions - und Treuhand AG im Zentralen Staatsarchiv Potsdam, Historische Abtellung I

BIBLIOGRA PHIE

Bibliographie wirtschaftsgeschichtlicher Literatur der DDR, 4. Lieferung 
